\title{
Therapeutic drug monitoring of once daily aminoglycoside dosing: comparison of two methods and investigation of the optimal blood sampling strategy
}

\author{
Lana Nezic • Adrian Derungs • Marcel Bruggisser • \\ Sarah Tschudin-Sutter • Stephan Krähenbühl • \\ Manuel Haschke
}

Received: 27 December 2013 / Accepted: 3 April 2014 /Published online: 23 April 2014

(C) Springer-Verlag Berlin Heidelberg 2014

\begin{abstract}
Purpose Therapeutic drug monitoring of patients receiving once daily aminoglycoside therapy can be performed using pharmacokinetic (PK) formulas or Bayesian calculations. While these methods produced comparable results, their performance has never been checked against full PK profiles. We performed a PK study in order to compare both methods and to determine the best time-points to estimate $\mathrm{AUC}_{0-24}$ and peak concentrations $\left(C_{\max }\right)$.

Methods We obtained full PK profiles in 14 patients receiving a once daily aminoglycoside therapy. PK parameters were calculated with PKSolver using non-compartmental methods. The calculated PK parameters were then compared with parameters estimated using an algorithm based on two serum concentrations (two-point method) or the software TCIWorks (Bayesian method).
\end{abstract}

Lana Nezic, Adrian Derungs, and Marcel Bruggisser contributed equally to this work.

Electronic supplementary material The online version of this article (doi:10.1007/s00228-014-1680-3) contains supplementary material, which is available to authorized users.

L. Nezic $\cdot$ A. Derungs $\cdot$ M. Bruggisser $\cdot$ S. Krähenbühl $(\varangle) \cdot$

M. Haschke

Division of Clinical Pharmacology and Toxicology, University

Hospital Basel, 4031 Basel, Switzerland

e-mail: stephan.kraehenbuehl@usb.ch

\section{A. Derungs}

University Clinic for Nephrology, Hypertension and Clinical

Pharmacology, University Hospital Bern, Bern, Switzerland

S. Krähenbühl · M. Haschke

Department of Biomedicine, University of Basel, Basel, Switzerland

S. Tschudin-Sutter

Division of Infectious Diseases and Hospital Epidemiology,

University Hospital Basel, Basel, Switzerland
Results For tobramycin and gentamicin, $\mathrm{AUC}_{0-24}$ and $C_{\max }$ could be reliably estimated using a first serum concentration obtained at $1 \mathrm{~h}$ and a second one between 8 and $10 \mathrm{~h}$ after start of the infusion. The two-point and the Bayesian method produced similar results. For amikacin, $\mathrm{AUC}_{0-24}$ could reliably be estimated by both methods. $C_{\max }$ was underestimated by $10-20 \%$ by the two-point method and by up to $30 \%$ with a large variation by the Bayesian method.

Conclusions The ideal time-points for therapeutic drug monitoring of once daily administered aminoglycosides are $1 \mathrm{~h}$ after start of a 30-min infusion for the first time-point and 8$10 \mathrm{~h}$ after start of the infusion for the second time-point. Duration of the infusion and accurate registration of the time-points of blood drawing are essential for obtaining precise predictions.

Keywords Gentamicin · Tobramycin · Amikacin . Once-daily dosing $\cdot$ Pharmacokinetics

\section{Introduction}

Aminoglycosides were among the first antibiotics introduced into clinics [1] and are still used widely, mainly in the treatment of severe gram-negative infections [2]. Rapid concentration-dependent killing, low prevalence of bacterial resistance, a marked post-antibiotic effect, and low costs are the main causes for their continued use [2, 3]. During the last two decades, extended dosing interval strategies, in particular, once-daily dosing (ODD), are increasingly recommended and used [4]. The possible therapeutic advantage of aminoglycoside ODD over conventional multiple daily dosing is a lower incidence of renal and ototoxicity with equal or better efficacy [5-9]. Importantly, the desired ratio of maximal drug concentration $\left(C_{\max }\right)$ over the minimal inhibitory concentration 
(MIC) of more than 8, which is needed for good efficacy of the aminoglycosides $[3,10]$, can be reached easily with ODD. Since aminoglycoside toxicity, in particular, nephrotoxicity, has been associated with increased trough concentrations $\left(C_{\min }\right)$ and duration of treatment $[11,12]$, ODD may also help to decrease aminoglycoside toxicity. Further advantages of aminoglycoside ODD include straightforward dosage calculation, decreased personnel time, possibly fewer assays for therapeutic drug monitoring (TDM), and, as a result, lower costs [13]. However, although ODD has been assessed in many clinical trials, there has been little uniformity regarding the best dosing and monitoring strategy for ODD. TDM based on individual pharmacokinetic values can reduce aminoglycoside toxicity and at the same time allow the administration of a larger cumulated dose [5].

The traditional concept of $C_{\max }$ and $C_{\min }$ measurement may not be appropriate for ODD of aminoglycosides for several reasons. In patients with normal renal function, the serum concentrations at $24 \mathrm{~h}$ usually are below the lower limit of quantification (LLOQ) of the routinely used immunoassays $[13,14]$. Furthermore, measurement of trough concentrations would delay treatment adjustments for at least one dose interval [15]. Measurement of the peak concentration ensures that the $C_{\max }$ is sufficiently above the MIC of the pathogen and is therefore important to judge the efficacy of the treatment. On the other hand, $C_{\max }$ is not an ideal marker for toxicity, which is best reflected by the area under the curve (AUC) and the trough serum concentration [16].

Taking into account these considerations, Begg et al. [13, 14] proposed a method of aminoglycoside ODD drug monitoring based on a target AUC, which is equivalent to the AUC achieved in adult patients with conventional multiple daily dose regimens over $24 \mathrm{~h}$. They proposed to determine two serum concentrations, the first at approximately $0.5 \mathrm{~h}$ after the end of the infusion and the second at least 2.5 but not longer than five expected half-lives after the application. After estimation of $\mathrm{AUC}_{0-24}$ from these two values assuming onecompartment pharmacokinetics, dose adjustment is based on the ratio of calculated to target AUC using linear extrapolation $[13,14]$. The suitability of this method has been investigated in approximately 100 patients; in this study, Begg's method was reported to be practical [17]. With some limitations, this method can also be used for aminoglycoside dosing in patients with mild to moderate renal dysfunction. In patients with moderate to severe renal dysfunction, it may be necessary to increase the dose interval to more than $24 \mathrm{~h}$ in order to reach sufficiently high peak serum concentrations and avoid accumulation. However, dose intervals longer than $24 \mathrm{~h}$ have so far not been assessed in clinical trials.

An alternative method to Begg's method is the Bayesian approach [18], which, however, requires computation assistance. The comparability of the Bayesian method and Begg's method has recently been investigated and published for gentamicin by Wong et al. [19]. However, to our knowledge, so far, neither Begg's method nor the Bayesian method has been checked against full pharmacokinetic (PK) profiles.

We therefore obtained full $\mathrm{PK}$ profiles in 14 patients treated once daily with an aminoglycoside, allowing us to address the following questions: (1) How reliably can $C_{\max }$ and $\mathrm{AUC}_{0-24}$ of once-daily dosed aminoglycosides be estimated using mathematical models (i.e., Begg's algorithm [two-point method] or a Bayesian method) in comparison to determinations using full PK profiles? (2) Which time-points of the kinetic profile provide the best estimate for $C_{\max }$ and $\mathrm{AUC}_{0-24}$ ?

\section{Methods}

\section{Study design}

Fourteen patients with once-daily aminoglycoside dosing and normal or impaired (but stable) renal function over the last 3 days were prospectively included at the University Hospital of Basel. Aminoglycosides were dosed according to clinical routine. Patients with tobramycin therapy received $5-8 \mathrm{mg} / \mathrm{kg}$ bodyweight and patients with gentamicin therapy $2-3 \mathrm{mg} / \mathrm{kg}$ bodyweight. Patients with amikacin (mostly patients with febrile neutropenia) were treated according to an internal protocol with a starting dose of $1,000 \mathrm{mg}$ amikacin. Aminoglycosides were diluted in normal saline and infused intravenously over $30 \mathrm{~min}$. Blood samples (serum) for the PK profiles were collected at the following time-points: $t_{\text {start }}=$ before start of the aminoglycoside infusion (baseline), $t_{\mathrm{end}}=$ at end of aminoglycoside infusion (30 $\mathrm{min}$ ) and $45 \mathrm{~min}, 1,2,4,6,8,12$, and $24 \mathrm{~h}$ after the start of the infusion. Blood samples were either obtained by a peripheral intravenous line placed at the contralateral side of the aminoglycoside infusion or by an aminoglycoside free lumen of a central venous line. The study was approved by the Ethics Committee of Basel and was conducted in accordance with the Declaration of Helsinki and the International Conference on Harmonization Good Clinical Practice guidelines. All patients gave written informed consent.

\section{Analytics}

Aminoglycoside serum levels were measured by the Clinical Chemistry laboratory of the University Hospital Basel using different commercially available methods. Amikacin was measured using the Roche Kinetic Interaction of Microparticles in Solution assay AMIK2, which has a LLOQ of $0.8 \mathrm{mg} /$ L. Tobramycin was measured using the Roche Enzyme Multiplied Immunoassay Technique assay TOBR2 with an LLOQ of $0.33 \mathrm{mg} / \mathrm{L}$. The assays for gentamicin had changed during the study period. From May 2010 until August 2010, gentamicin was measured using a fluorescence polarization 
immunoassay (Abbott, USA, LLOQ $0.3 \mathrm{mg} / \mathrm{L}$ ). From September 2010 until July 2011, gentamicin was measured using a cloned enzyme donor immunoassay (Thermofisher, USA, LLOQ, $0.3 \mathrm{mg} / \mathrm{L}$ ). From July 2011 until December 2011, gentamicin was measured using a chemiluminescence microparticle assay (Abbott, USA, LLOQ $0.3 \mathrm{mg} / \mathrm{L}$ ).

Pharmacokinetic analysis

PK parameters obtained from full PK profiles (labeled as $X^{\text {obs }}$ )

Serum concentration data were analyzed using the Excel add-in PKSolver from Zhang et al. [20]. For the calculation of the AUC from time of dosing to the last observable concentration $\left(\mathrm{AUC}_{0 \text {-last }}\right.$ obs $)$, the "mixed linear up/log down" model was chosen. As the last observable concentration $\left(C_{\text {last }}\right.$ obs $)$ was not always identical to the concentration at $24 \mathrm{~h}\left(C_{24}{ }^{\text {obs }}\right)$, the latter had to be calculated as follows (Eq. 1):

$C_{24}^{\mathrm{obs}}=C_{\mathrm{last}}^{\mathrm{obs}} \cdot e^{\left[-k_{e}^{\mathrm{obs}} \cdot\left(t_{24}-t_{\text {last }}\right)\right]}$

where $k_{\mathrm{e}}{ }^{\text {obs }}$ was the elimination rate constant, given by $P K S o l v e r$. AUC from 0 to $24 \mathrm{~h}$ was then calculated as follows (Eq. 2):

$\mathrm{AUC}_{0-24}^{\mathrm{obs}}=\mathrm{AUC}_{0-\text { last }}^{\mathrm{obs}}+\frac{C_{\text {last }}^{\mathrm{obs}}-C_{24}^{\mathrm{obs}}}{\ln \left(\mathrm{C}_{\text {last }}^{\mathrm{obs}}\right)-\ln \left(\mathrm{C}_{24}^{\mathrm{obs}}\right)}\left(t_{24}-t_{\text {last }}\right)$

$T_{\max }{ }^{\text {obs }}$ and $C_{\max }{ }^{\text {obs }}$ were obtained directly from observed concentration-time data.

$P K$ parameters obtained by two-point estimation (labeled as $X^{2-p o i n t}$ )

PK parameters were calculated using two concentration-time point pairs $\left(t_{1} / C_{1}, t_{2} / C_{2}\right)$ according to the algorithm proposed by Begg et al. [13] with a modification for $\mathrm{AUC}_{0-24}$. The estimated elimination rate constant $\left(k_{e}^{2 \text {-point }}\right)$ is given by (Eq. 3):

$k_{e}^{2 \text {-point }}=\frac{\ln \left(C_{1}\right)-\ln \left(C_{2}\right)}{t_{2}-t_{1}}$

With this, the concentration at end of infusion $\left(C_{\text {end }}{ }^{2 \text {-point }}\right)$ is (Eq. 4):

$C_{\text {end }}^{2 \text {-point }}=C_{1} \cdot e^{\left[-k_{e}^{2 \text {-point }} \cdot\left(t_{\text {end }}-t_{1}\right)\right]}$ where $t_{\text {end }}$ is the infusion time. The concentration at the end of the dosing interval $\left(C_{24}{ }^{2 \text {-point }}\right)$ is (Eq. 5):

$C_{24}^{2 \text {-point }}=C_{2} \cdot e^{\left[-k_{e}^{2-\text { point }}\left(t_{24}-t_{2}\right)\right]}$

With this, the $\mathrm{AUC}_{0-24}{ }^{2 \text {-point }}$ was calculated as follows (Eq. 6):

$\mathrm{AUC}_{0-24}^{2 \text {-point }}=\mathrm{AUC}_{0-\text { end }}+\mathrm{AUC}_{\text {end-24 }}=\frac{C_{\text {end }}^{2 \text {-point }} \cdot t_{\text {end }}}{2}+\frac{C_{\text {end }}^{2 \text {-point }}-C_{24}^{2 \text {-point }}}{k_{e}^{2 \text {-point }}}$

While we prefer to calculate $\mathrm{AUC}_{0 \text {-end, }}$, Begg et al. assumed that $\mathrm{AUC}_{0 \text {-end }}$ was $6.5 \%$ of $\mathrm{AUC}_{\text {end-24 }}$ [13].

These PK parameters were estimated using different timepoint combinations $(1$ and $6 \mathrm{~h}, 1$ and $8 \mathrm{~h}, 1$ and $12 \mathrm{~h}, 2$ and $6 \mathrm{~h}$, 2 and $8 \mathrm{~h}, 2$ and $12 \mathrm{~h}$ ), and the estimates $\left(X^{2 \text {-point }}\right)$ were then compared with the calculated parameters based on full PK profiles $\left(X^{\mathrm{obs}}\right)$.

$P K$ parameters obtained by Bayesian estimation (labeled as $X^{T C I}$ )

Bayesian analysis was performed using the software package TCIWorks (freely available at www.tciworks.info). For tobramycin and gentamicin, the built-in model "Gentamicin/ Tobramycin" was used, and for amikacin, the model "Amikacin for Adults". Both models were used in the onecompartment mode. $\mathrm{AUC}_{0-24}{ }^{\mathrm{TCI}}$ and $C_{\max }{ }^{\mathrm{TCI}}$ were determined using the same time-point combinations as for the Excel-based two-point estimation (i.e., 1 and $6 \mathrm{~h}, 1$ and $8 \mathrm{~h}$, 1 and 12 h, 2 and 6 h, 2 and 8 h, 2 and 12 h). The estimates $\left(X^{\mathrm{TCI}}\right)$ were then compared with the PK parameters based on full PK profiles $\left(X^{\mathrm{obs}}\right)$.

Graphical and statistical analyses

Figures were drawn with GraphPad Prism 6.02 for Windows. Due to the small sample size, statistical significance testing was not performed.

\section{Results}

The baseline characteristics of the 14 patients are shown in Table 1. Seven patients were treated with tobramycin, three with gentamicin, and four with amikacin; all of them were managed using Begg's algorithm. Five patients were clinically and microbiologically cured and could leave the hospital, whereas nine improved clinically and could be transferred to 
Table 1 Baseline characteristics of the patients included in the study

\begin{tabular}{|c|c|c|c|c|c|c|c|c|c|}
\hline ID & $\begin{array}{l}\text { Age } \\
\text { [years] }\end{array}$ & Sex & $\begin{array}{l}\text { Weight } \\
{[\mathrm{kg}]}\end{array}$ & $\begin{array}{l}\text { eGFR }[\mathrm{ml} / \mathrm{min} / \\
\left.1.73 \mathrm{~m}^{2}\right]\end{array}$ & Therapy & $\begin{array}{l}\text { Dose } \\
{[\mathrm{mg}]}\end{array}$ & $\begin{array}{l}\text { Dose per kilogram } \\
{[\mathrm{mg} / \mathrm{kg}]}\end{array}$ & $\begin{array}{l}\text { Infusion } \\
\text { time [min] }\end{array}$ & Diagnosis/indication \\
\hline 101 & 61 & $\mathrm{f}$ & 60 & 106 & Tobramycin & 380 & 6.3 & 45 & $\begin{array}{l}\text { Osteomyelitis; Pseudomonas aeruginosa, } \\
\text { Serratia liquefaciens }\end{array}$ \\
\hline 102 & 73 & $\mathrm{~m}$ & 73 & 98 & Tobramycin & 560 & 7.7 & 35 & Septic gonarthritis; $P$. aeruginosa \\
\hline 103 & 28 & $\mathrm{~m}$ & 62 & 121 & Tobramycin & 420 & 6.8 & 35 & Cystic fibrosis; $P$. aeruginosa, ESBL \\
\hline 105 & 65 & $\mathrm{~m}$ & 80 & 72 & Tobramycin & 500 & 6.3 & 30 & $\begin{array}{l}\text { Pleural empyema; } P \text {. aeruginosa, Klebsiella } \\
\text { pneumoniae }\end{array}$ \\
\hline 108 & 49 & $\mathrm{~m}$ & 82 & 109 & Tobramycin & 570 & 7.0 & 30 & Spondylodiscitis; $P$. aeruginosa \\
\hline 112 & 74 & $\mathrm{~m}$ & 90 & 129 & Tobramycin & 460 & 5.1 & 30 & $\begin{array}{l}\text { Wound infection after laminectomy; } \\
\text { P. aeruginosa }\end{array}$ \\
\hline 116 & 23 & $\mathrm{f}$ & 50 & 129 & Tobramycin & 280 & 5.6 & 32 & Cystic fibrosis; $P$. aeruginosa \\
\hline 104 & 69 & $\mathrm{~m}$ & 80 & 95 & Gentamicin & 240 & 3.0 & 30 & $\begin{array}{l}\text { Spondylodiscitis, epidural abscess; } \\
\text { Streptococcus mitis/oralis }\end{array}$ \\
\hline 109 & 67 & $\mathrm{f}$ & 68 & 96 & Gentamicin & 200 & 2.9 & 30 & $\begin{array}{l}\text { Aortic valve prosthesis endocarditis; } \\
\text { Staphylococcus epidermidis }\end{array}$ \\
\hline 114 & 61 & $\mathrm{~m}$ & 110 & 92 & Gentamicin & 240 & 2.2 & 30 & Aortic valve endocarditis \\
\hline 107 & 39 & $\mathrm{~m}$ & 85 & 136 & Amikacin & 1,000 & 11.8 & 30 & neutropenic fever \\
\hline 110 & 28 & $\mathrm{f}$ & 48 & 123 & Amikacin & 1,000 & 20.8 & 30 & $\begin{array}{l}\text { Tuberculosis; Mycobacterium tuberculosis } \\
\text { complex }\end{array}$ \\
\hline 111 & 26 & $\mathrm{~m}$ & 94 & 117 & Amikacin & 1,000 & 10.6 & 25 & Neutropenic fever \\
\hline 115 & 42 & $\mathrm{~m}$ & 72 & 105 & Amikacin & 1,000 & 13.9 & 30 & Neutropenic fever \\
\hline
\end{tabular}

another hospital. None of the patients developed a significant change in renal function during the hospitalization. With the exception of patient 105 (see ID number in Table 1), the estimated GFR was $>90 \mathrm{ml} / \mathrm{min} / 1.73 \mathrm{~m}^{2}$ (CKD-EPI formula). Thus, our results can be generalized only to patients with normal renal function.

Figure 1 shows the PK profiles according to the administered aminoglycoside. As can be seen, most semi-logarithmic plots are not linear. Some profiles show a bi-exponential shape, and some appear to be even tri-exponential. For Bayesian analyses, this could be taken into account by choosing an appropriate model. In contrast, the two-point estimation according to the algorithm proposed by Begg et al. is based on a one-compartment model with linear elimination [13]. It could therefore be expected that, in the case of one compartment models, the estimation of $C_{\max }$ and AUC is going to depend on the two time-points chosen for their calculation.

Figure 2 shows the accuracy of the two-point estimation and the Bayesian estimation (TCI method) for different timepoint combinations for $C_{\max }$. The values shown in the figure are the ratios of the calculated and the corresponding observed values.

For tobramycin and gentamicin, the mean estimates were $8 \%$ to $26 \%$ lower than the corresponding observed values for all time point combinations. The differences between the twopoint and Bayesian estimations were only small. The variation tended to be more accentuated for the two-point estimation than for the Bayesian method and became larger when the first determination was shifted from 1 to $2 \mathrm{~h}$ after start of the infusion.

For amikacin, the mean estimates were 15 to $35 \%$ lower than the observed values with an acceptable variability for the two-point method estimates, whereas the variability was much higher for the Bayesian estimates. The large variability for the Bayesian estimates was mainly due to patient 107, who had ratios between 1.72 (for $1 / 6 \mathrm{~h}$ ) and 2.05 (for $2 / 6 \mathrm{~h}$ ). Patient 107 had the highest eGFR, resulting in a high elimination rate and therefore a high estimate of $C_{\max }$ with the Bayesian method.

The AUC estimations showed a different picture compared with the estimations for $C_{\max }$ (Fig. 3). For all three drugs, the estimated $\mathrm{AUC}_{0-24}$ increased with the interval between the two time-points. In contrast to the estimations for $C_{\max }$, the choice of the first-time-point ( $1 \mathrm{vs} .2 \mathrm{~h}$ ) was hardly relevant for the estimation of the $\mathrm{AUC}_{0-24}$. The accuracy of the $\mathrm{AUC}_{0-24}$ estimation depended on the time-point of the second measurement and the time interval between the two measurements. Considering tobramycin, the time-point combinations $1 / 12 \mathrm{~h}$ and $2 / 12 \mathrm{~h}$ were ideal for the two-point method. For the Bayesian estimation, the combination $1 / 12 \mathrm{~h}$ was slightly superior compared with the combination $2 / 12 \mathrm{~h}$. For gentamicin, the combinations $1 / 8 \mathrm{~h}$ and $2 / 12 \mathrm{~h}$ were best for both calculation methods. For amikacin, the combinations $1 / 8 \mathrm{~h}$, $2 / 8 \mathrm{~h}$, and $2 / 12 \mathrm{~h}$ gave the best results for both calculation methods, but, similar to $C_{\max }$, the values for $\mathrm{AUC}_{0-24}$ showed 

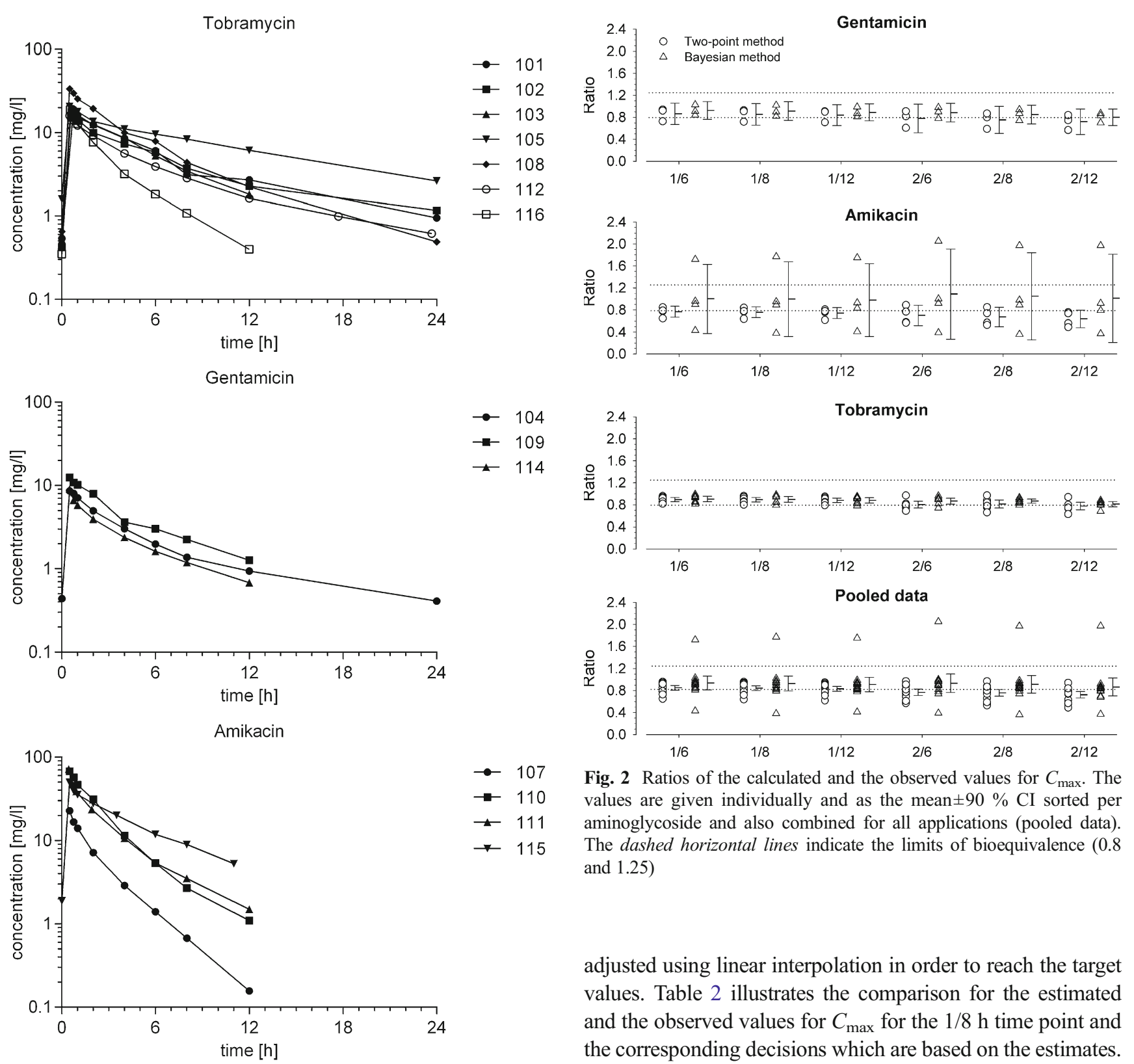

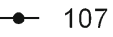

$\rightarrow-110$

$\leftarrow 111$

$\rightarrow \quad 115$

Fig. 1 Semilogarithmic plots of the PK profiles of the 14 patients sorted by the aminoglycoside administered. Most profiles are not linear and show a distribution and one to two elimination phases

a large variation for the Bayesian estimates. Considering only the mean values, the differences between the two calculation methods were small.

In the clinical situation, the exact difference between the estimated and the observed values is less relevant. The physician would rather like to know whether the treatment decision, which is based on the described calculations, was correct. If the estimated $C_{\max }$ is within the target range (i.e., $15-25 \mathrm{mg} / \mathrm{L}$ for tobramycin and gentamicin; 55-65 mg/L for amikacin) and the AUC does not exceed the upper limit $(100 \mathrm{mg} / \mathrm{L} * \mathrm{~h}$ for gentamicin and tobramycin and $240 \mathrm{mg} / \mathrm{L} * \mathrm{~h}$ for amikacin), no dose adjustment is recommended. Otherwise, the dose is

Fig. 2 Ratios of the calculated and the observed values for $C_{\max }$. The values are given individually and as the mean $\pm 90 \%$ CI sorted per aminoglycoside and also combined for all applications (pooled data). The dashed horizontal lines indicate the limits of bioequivalence $(0.8$ and 1.25)

adjusted using linear interpolation in order to reach the target values. Table 2 illustrates the comparison for the estimated and the observed values for $C_{\max }$ for the $1 / 8 \mathrm{~h}$ time point and the corresponding decisions which are based on the estimates. The decisions are labeled "wrong" when another decision would have been made based on the observed value. For the two-point estimations, the decisions would have been correct with the exception of patients 110 and 112. In patient 112, the tobramycin dose would have been increased instead of maintained and in patient 110 , the amikacin dose would have been maintained instead of decreased. For the Bayesian estimation, the decisions would have been correct except for patients 102 and 110. For patient 102, the dose would have been increased instead of maintained and for patient 110, the dose would have been maintained instead of decreased. As shown in Electronic supplementary material Table 1, the combination 01/06 h showed a comparable performance like the combination $01 / 08 \mathrm{~h}$, whereas the performance of the other combinations $(01 / 12 \mathrm{~h}, 02 / 06 \mathrm{~h}, 02 / 08 \mathrm{~h}, 02 / 12 \mathrm{~h})$ was worse for both calculation methods. 
Fig. 3 Ratios of the calculated and the observed values for $\mathrm{AUC}_{0-24}$. The values are given individually and as the mean \pm $90 \%$ CI sorted per aminoglycoside and also combined for all applications (pooled data). The dashed horizontal lines indicate the limits of bioequivalence ( 0.8 and 1.25$)$
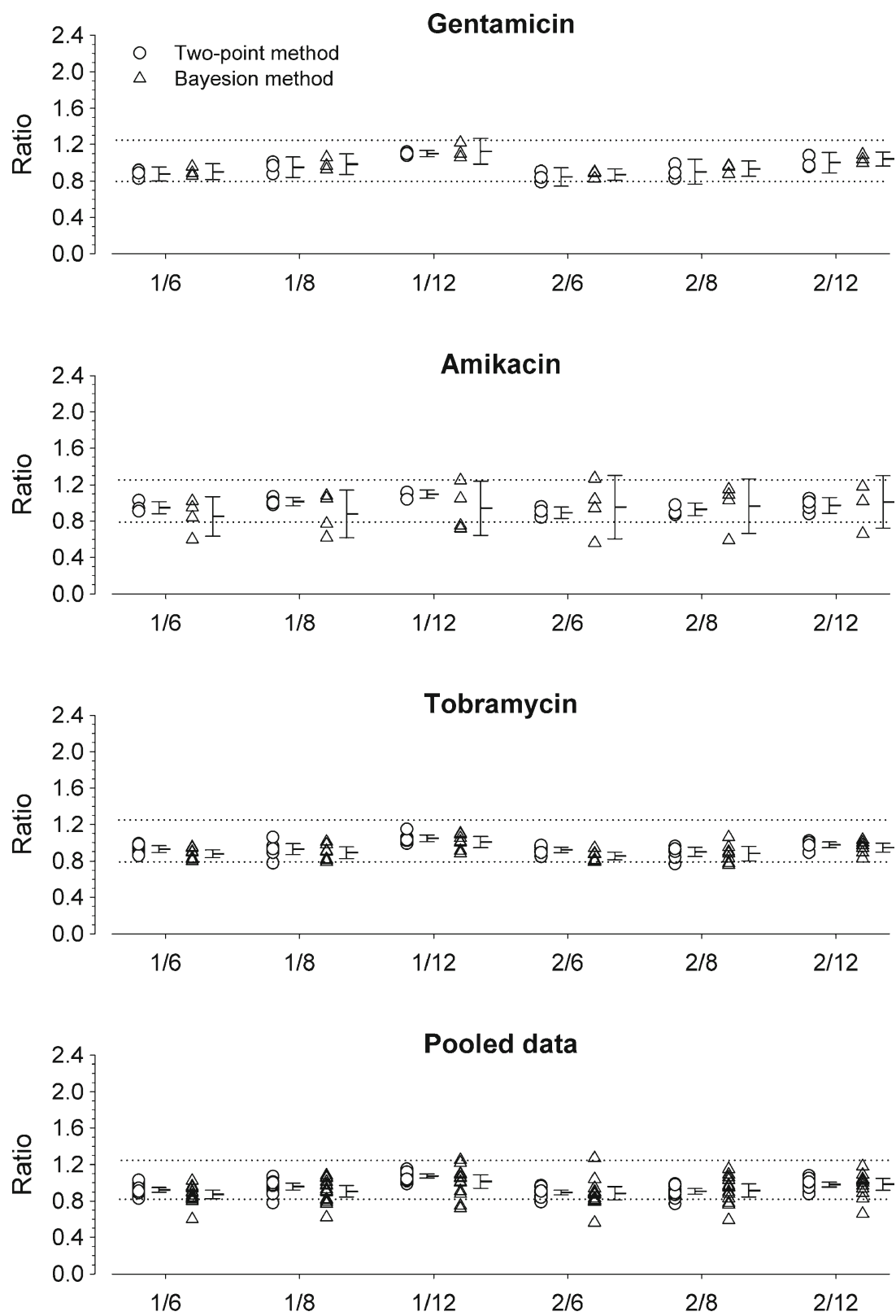

Table 3 illustrates the comparison for the estimated and the observed values for $\mathrm{AUC}_{0-24}$ for the 1/8 h time point and the corresponding decisions which are based on the estimates. For the two-point method, wrong decisions would have been made for patients 101 and 112. For patient 101, the tobramycin dose would have been maintained instead of reduced and for patient 112 , the tobramycin dose would have been increased instead of maintained. For the Bayesian estimation, only for patient 101 would a wrong decision (tobramycin dose maintained instead of reduced) have been made. The comparisons between the estimated and observed values for $\mathrm{AUC}_{0-24}$ for the other time-point combinations are shown in Electronic supplementary material Table 2. As expected, the best performance was achieved for the time points with the longest interval between the measurements $(1 / 12 \mathrm{~h}$ and $2 / 12 \mathrm{~h}$ ). The time points $1 / 8 \mathrm{~h}$ and $2 / 8 \mathrm{~h}$ were considered to have an acceptable performance, whereas the error rate for the time points $1 / 6 \mathrm{~h}$ and $2 / 6 \mathrm{~h}$ were considered to be too high.

\section{Discussion}

Our study shows that $C_{\max }$ and $\mathrm{AUC}_{0-24}$ of once-daily dosed aminoglycosides can be estimated reliably with a two-point mono-compartmental method but that the results obtained depend on the time-points chosen for the concentration measurements. For the estimation of $C_{\max }$, the best time-point combinations were $1 / 6-8 \mathrm{~h}$ and for $\mathrm{AUC}_{0-24} 1-2 / 8-12 \mathrm{~h}$ after 
Table 2 Comparison of the estimated and observed values for $C_{\max }$ for the time point combination $1 / 8 \mathrm{~h}$

\begin{tabular}{|c|c|c|c|c|c|c|c|c|}
\hline ID & Therapy & $\begin{array}{l}\text { Dose } \\
{[\mathrm{mg} / 24 \mathrm{~h}]}\end{array}$ & $\begin{array}{l}\text { Target } C_{\max } \\
{[\mathrm{mg} / \mathrm{L}]}\end{array}$ & $\begin{array}{l}C_{\max } \text { observed } \\
{[\mathrm{mg} / \mathrm{L}]}\end{array}$ & $\begin{array}{l}C_{\max }^{2-\text { point }} \\
{[\mathrm{mg} / \mathrm{L}]}\end{array}$ & Decision & $\begin{array}{l}C_{\max }{ }^{\mathrm{TCI}} \\
{[\mathrm{mg} / \mathrm{L}]}\end{array}$ & Decision \\
\hline 101 & Tobramycin & 380 & $15-25$ & 19.0 & 17.2 & Correct & 17.9 & Correct \\
\hline 102 & Tobramycin & 560 & $15-25$ & 15.8 & 15.2 & Correct & 14.9 & Wrong \\
\hline 103 & Tobramycin & 420 & $15-25$ & 17.7 & 16.6 & Correct & 15.1 & Correct \\
\hline 105 & Tobramycin & 500 & $15-25$ & 20.8 & 19.0 & Correct & 20.3 & Correct \\
\hline 108 & Tobramycin & 570 & $15-25$ & 33.5 & 28.8 & Correct & 28.2 & Correct \\
\hline 112 & Tobramycin & 460 & $15-25$ & 16.0 & 13.7 & Wrong & 15.2 & Correct \\
\hline 116 & Tobramycin & 280 & $15-25$ & 19.0 & 15.2 & Correct & 15.0 & Correct \\
\hline 104 & Gentamicin & 240 & $15-25$ & 8.6 & 8.0 & Correct & 8.8 & Correct \\
\hline 109 & Gentamicin & 200 & $15-25$ & 12.5 & 11.3 & Correct & 11.2 & Correct \\
\hline 114 & Gentamicin & 240 & $15-25$ & 9.1 & 6.5 & Correct & 7.5 & Correct \\
\hline 107 & Amikacin & 1,000 & $55-65$ & 22.8 & & & & \\
\hline 110 & Amikacin & 1,000 & $55-65$ & 67.9 & 57.1 & Wrong & 64.6 & Wrong \\
\hline 111 & Amikacin & 1,000 & $55-65$ & 72.2 & & & & \\
\hline 115 & Amikacin & 1,000 & $55-65$ & 50.0 & 39.2 & Correct & 44.6 & Correct \\
\hline
\end{tabular}

Values for $C_{\max }$ were estimated using either the two-point method $\left(C_{\max }{ }^{2-p o i n t}\right)$ or the Bayesian method $\left(C_{\max }{ }^{\text {TCI }}\right)$ as described in Methods. Based on these estimates, decisions were made according to the target values. If the decisions made based on the estimated values corresponded to those made using the observed values, they were termed "correct". If there was a disagreement between the decisions based on the estimated and the observed values, they were termed "wrong"

start of the infusion. The accuracy of the prediction was similar for the two-point and for the Bayesian method (target concentration intervention software TCIWorks).

Using two (or more) serum drug concentrations to determine the next aminoglycoside dose has already been proposed more than 10 years ago by Sawchuck and Zaske [21] as well as by Begg et al. [13]. The usually recommended procedure is to measure the aminoglycoside plasma concentration at two time-points, the first up to $4 \mathrm{~h}$ after the end of the infusion and the second $6-14 \mathrm{~h}$ after the end of the infusion [19]. The current study shows that $C_{\max }$ can be estimated more accurately, if the first time-point is chosen early. On the other hand,

Table 3 Comparison of the estimated and observed values for $\mathrm{AUC}_{0-24}$ for the time point combination $1 / 8 \mathrm{~h}$

\begin{tabular}{|c|c|c|c|c|c|c|c|c|}
\hline ID & Therapy & $\begin{array}{l}\text { Dose } \\
{[\mathrm{mg} /} \\
24 \mathrm{~h}]\end{array}$ & $\begin{array}{l}\text { Target AUC } \mathrm{A}_{0-24} \\
{[\mathrm{mg} / \mathrm{L} * \mathrm{~h}]}\end{array}$ & $\begin{array}{l}\mathrm{AUC}_{0-24} \text { observed } \\
{[\mathrm{mg} / \mathrm{L} * \mathrm{~h}]}\end{array}$ & $\begin{array}{l}\mathrm{AUC}_{0-24}{ }^{2 \text {-point }} \\
{[\mathrm{mg} / \mathrm{L} * \mathrm{~h}]}\end{array}$ & Decision & $\begin{array}{l}\mathrm{AUC}_{0-24}{ }^{\mathrm{TCI}} \\
{[\mathrm{mg} / \mathrm{L} * \mathrm{~h}]}\end{array}$ & Decision \\
\hline 101 & Tobramycin & 380 & $70-100$ & 102 & 79.5 & Wrong & 81 & Wrong \\
\hline 102 & Tobramycin & 560 & 70-100 & 95 & 84.1 & Correct & 77 & Correct \\
\hline 103 & Tobramycin & 420 & $70-100$ & 88 & 82.7 & Correct & 72 & Correct \\
\hline 105 & Tobramycin & 500 & $70-100$ & 173 & 165.2 & Correct & 157 & Correct \\
\hline 108 & Tobramycin & 570 & $70-100$ & 131 & 122.1 & Correct & 118 & Correct \\
\hline 112 & Tobramycin & 460 & $70-100$ & 74 & 68.4 & Wrong & 72 & Correct \\
\hline 116 & Tobramycin & 280 & $70-100$ & 44 & 46.9 & Correct & 45 & Correct \\
\hline 104 & Gentamicin & 240 & $70-100$ & 41 & 36.1 & Correct & 38 & Correct \\
\hline 109 & Gentamicin & 200 & $70-100$ & 55 & 55.2 & Correct & 53 & Correct \\
\hline 114 & Gentamicin & 240 & $70-100$ & 31 & 30.3 & Correct & 33 & Correct \\
\hline 107 & Amikacin & 1,000 & $200-240$ & 42 & & & & \\
\hline 110 & Amikacin & 1,000 & $200-240$ & 159 & 154.6 & Correct & 171 & Correct \\
\hline 111 & Amikacin & 1,000 & $200-240$ & 144 & & & & \\
\hline 115 & Amikacin & 1,000 & $200-240$ & 208 & 207.5 & Correct & 218 & Correct \\
\hline
\end{tabular}

Values for $\mathrm{AUC}_{0-24}$ were estimated using either the two-point method ( $\mathrm{AUC}_{0-24}{ }^{2 \text {-point }}$ ) or the Bayesian method ( $\mathrm{AUC} \mathrm{C}_{0-24}{ }^{\mathrm{TCI}}$ ) as described in Methods. Based on these estimates, decisions were made according to the target values. If the decisions made based on the estimated values corresponded to those made using the observed values, they were termed "correct". If there was a disagreement between the decisions based on the estimated and the observed values, they were termed "wrong" 
the prediction of $\mathrm{AUC}_{0-24}$ depends more on the duration of the interval between the two time-points. Accordingly, the best time-points for the estimation of $C_{\max }$ were $1 / 6-8 \mathrm{~h}$ and for $\mathrm{AUC}_{0-24} 1-2 / 8-12 \mathrm{~h}$. Therefore, we propose to obtain the first plasma sample $1 \mathrm{~h}$ after start of the infusion (duration of the infusion $30 \mathrm{~min}$ ) and the second one $8-10 \mathrm{~h}$ after start of the infusion. Considering the typical half-life of $2-4 \mathrm{~h}$ for all aminoglycosides, the second plasma sample would be obtained two to five half-lives after the start of the infusion. This is consistent with Begg's proposal, who recommended the determination of aminoglycoside concentrations $0.5 \mathrm{~h}$ after the end of the infusion and between 2.5 and 5 half-lives later, whereby the infusion should be administered within $30 \mathrm{~min}$ [13].

With our proposal considering the time-points of blood sampling (1/8-10 h), the mean error of the $\mathrm{AUC}_{0-24}$ calculated with the two-point method does not exceed $\pm 10 \%$ regardless of the aminoglycoside administered, which appears to be acceptable. The mean peak concentrations for tobramycin and gentamicin underestimated the observed values by 10 $20 \%$, which is also tolerable in our opinion. For amikacin, the two-point method underestimated the mean $C_{\max }$ by $22 \%$, which may be due to the short half-life of this drug (Fig. 1). The underestimation of $C_{\max }$ by the two-point method can partially be explained by the observation that all aminoglycosides investigated showed a considerable distribution phase lasting 1 to $2 \mathrm{~h}$ after termination of the infusion (Fig. 1). Since it can be questioned whether the distribution phase contributes to the antibacterial activity of the aminoglycosides, we propose to accept the calculated value at the end of the infusion as $C_{\max }$, even if it underestimates the observed $C_{\max }$ by up to $22 \%$. Since $C_{\max }$ is related to efficacy (and not to toxicity if $\mathrm{AUC}_{0-24}$ is within the target range), this approach guarantees efficacy while minimizing toxicity.

An alternative method to the Excel method is the Bayesian approach [18]. Bayesian methods are currently considered to be the gold standard in therapeutic drug monitoring, but they require computational assistance and are usually based on pharmacokinetic data originating from different populations than the individual patients studied. There are different computer tools available to assist clinicians for Bayesian calculations for aminoglycosides [22]. One of these computer tools is the target concentration intervention software package TCIWorks, which is running under the Windows operating system. The program is based on models from results of previous pharmacokinetic studies and utilizes the creatinine clearance $\left(\mathrm{Cl}_{\mathrm{Cr}}\right)$ as a surrogate of the aminoglycoside clearance, since the systemic clearance of aminoglycosides correlates with $\mathrm{Cl}_{\mathrm{Cr}}$ [19].

The comparability of the Bayesian method (TCI method) with the two-point method has recently been investigated and demonstrated for gentamicin by Wong et al. [19]. Our results are consistent with those obtained by Wong et al., although our study population was smaller than Wong's. The current study shows that the results of the two-point and the Bayesian method are comparable also for tobramycin. Regarding amikacin, the TCI method tended to be less accurate, which was mainly due to one patient with an exceptionally high $\mathrm{Cl}_{\mathrm{Cr}}$. Regarding amikacin, more data are therefore necessary to compare the two methods for dose prediction.

It is important to realize that the time-points (start and end of the infusion, withdrawal of blood samples) have to be determined accurately; otherwise, the calculations can become erroneous. Much effort has therefore to be made that these time-points are recorded and transmitted accurately. An important point in this respect is the choice of the application time, which determines the time-points when the two blood samples have to be drawn. In our experience, best results are obtained when the two blood samples are obtained during the day shift and not during the night. If clinically possible, we therefore advise to administer aminoglycosides in the morning in order to obtain the second blood sample in the afternoon, allowing the calculation of the next dose still during the day shift. Another important point is the duration of the infusion, which should be $30 \mathrm{~min}$. Deviations in the duration of the infusion could also lead to erroneous calculations, especially considering $C_{\max }$.

The limited number of patients included is clearly a weakness of the study, particularly for amikacin. Another weakness is the fact that all patients included had a normal or only slightly reduced renal function and were not severely septic. The results of our study can therefore only be generalized to this patient population. Further studies are necessary for dose predictions of aminoglycosides in special populations, e.g., septic patients or patients with impaired renal function.

\section{Conclusion}

Our study indicates that the ideal time-points for therapeutic drug monitoring of once daily administered aminoglycosides are $1 \mathrm{~h}$ after start of a 30-min infusion for the first and 8-10 h after start of the infusion for the second blood sample. With these two time-points, $C_{\max }$ as well as $\mathrm{AUC}_{0-24}$ can be estimated reliably and can be used for determining the next dose, either by the simple two-point method using a onecompartment model or by the more sophisticated Bayesian approach.

Acknowledgments The authors would like to thank Claudia Bläsi, Luisa Baselgia-Jeker, and Beatrice Vetter for the excellent technical assistance.

Conflict of interest None of the authors reports any conflict of interest with this work. 
Funding source This study was supported by a grant from the Swiss National Science Foundation to SK (SNF 31003A-132992).

Contribution of the authors Lana Nezic: Prepared the first protocol, helped in the practical patient work, analyzed data, and approved the final version of the manuscript

Adrian Derungs: Helped in the practical patient work, analyzed data, helped to write the manuscript, and approved its final version

Marcel Bruggisser: Helped in preparing the final version of the protocol, helped in the practical patient work, and approved the final version of the manuscript

Sarah Tschudin-Sutter: Helped in preparing the final version of the protocol as an expert in infectiology and approved the final version of the manuscript

Stephan Krähenbühl: Approved the final version of the protocol, helped in writing the manuscript, and approved its final version

Manuel Haschke: Helped in preparing the final version of the protocol, analyzed data, helped in writing the manuscript, and approved its final version

\section{References}

1. Begg EJ, Barclay ML (1995) Aminoglycosides - 50 years on. Br J Clin Pharmacol 39(6):597-603

2. Avent ML, Rogers BA, Cheng AC, Paterson DL (2011) Current use of aminoglycosides: indications, pharmacokinetics and monitoring for toxicity. Intern Med J 41(6):441-449. doi:10.1111/j.1445-5994. 2011.02452.x

3. Pagkalis S, Mantadakis E, Mavros MN, Ammari C, Falagas ME (2011) Pharmacological considerations for the proper clinical use of aminoglycosides. Drugs 71(17):2277-2294. doi:10.2165/11597020000000000-00000

4. Hustinx WN, Hoepelman IM (1993) Aminoglycoside dosage regimens. Is once a day enough? Clin Pharmacokinet 25(6):427-432. doi:10.2165/00003088-199325060-00002

5. Bartal C, Danon A, Schlaeffer F, Reisenberg K, Alkan M, Smoliakov R, Sidi A, Almog Y (2003) Pharmacokinetic dosing of aminoglycosides: a controlled trial. Am J Med 114(3):194-198. doi:10.1016/ S0002-9343(02)01476-6

6. Barza M, Ioannidis JP, Cappelleri JC, Lau J (1996) Single or multiple daily doses of aminoglycosides: a meta-analysis. BMJ 312(7027): 338-345. doi:10.1136/bmj.312.7027.33

7. Hatala R, Dinh TT, Cook DJ (1997) Single daily dosing of aminoglycosides in immunocompromised adults: a systematic review. Clin Infect Dis 24(5):810-815. doi:10.1093/clinids/24.5.810

8. Mavros MN, Polyzos KA, Rafailidis PI, Falagas ME (2011) Once versus multiple daily dosing of aminoglycosides for patients with febrile neutropenia: a systematic review and meta-analysis. J Antimicrob Chemother 66(2):251-259. doi:10.1093/jac/dkq451
9. Munckhof WJ, Grayson ML, Turnidge JD (1996) A meta-analysis of studies on the safety and efficacy of aminoglycosides given either once daily or as divided doses. J Antimicrob Chemother 37(4):645663. doi:10.1093/jac/37.4.645

10. Turnidge J (2003) Pharmacodynamics and dosing of aminoglycosides. Infect Dis Clin North Am 17(3):503-528. doi:10.1016/S08915520(03)00057-6

11. Bertino JS Jr, Booker LA, Franck PA, Jenkins PL, Franck KR, Nafziger AN (1993) Incidence of and significant risk factors for aminoglycoside-associated nephrotoxicity in patients dosed by using individualized pharmacokinetic monitoring. J Infect Dis 167(1):173179. doi:10.1093/infdis/167.1.173

12. Croes S, Koop AH, van Gils SA, Neef C (2012) Efficacy, nephrotoxicity and ototoxicity of aminoglycosides, mathematically modelled for modelling-supported therapeutic drug monitoring. Eur J Pharm Sci 45(1-2):90-100. doi:10.1016/j.ejps.2011.10.022

13. Begg EJ, Barclay ML, Duffull SB (1995) A suggested approach to once-daily aminoglycoside dosing. Br J Clin Pharmacol 39(6):605609. doi:10.1111/j.1365-2125.1995.tb05719.x

14. Begg EJ, Barclay ML, Kirkpatrick CM (2001) The therapeutic monitoring of antimicrobial agents. Br J Clin Pharmacol 52(Suppl 1): 35S-43S. doi:10.1111/j.1365-2125.2001.00377.x

15. Barclay ML, Kirkpatrick CM, Begg EJ (1999) Once daily aminoglycoside therapy. Is it less toxic than multiple daily doses and how should it be monitored? Clin Pharmacokinet 36(2):89-98. doi:10. 2165/00003088-199936020-00001

16. Coulthard KP, Peckham DG, Conway SP, Smith CA, Bell J, Turnidge $\mathrm{J}$ (2007) Therapeutic drug monitoring of once daily tobramycin in cystic fibrosis - caution with trough concentrations. J Cyst Fibros 6(2):125-130. doi:10.1016/j.jcf.2006.05.015

17. Barclay ML, Duffull SB, Begg EJ, Buttimore RC (1995) Experience of once-daily aminoglycoside dosing using a target area under the concentration-time curve. Aust N Z J Med 25(3):230-235. doi:10. 1111/j.1445-5994.1995.tb01529.x

18. Burton ME, Brater DC, Chen PS, Day RB, Huber PJ, Vasko MR (1985) A Bayesian feedback method of aminoglycoside dosing. Clin Pharmacol Ther 37(3):349-357. doi:10.1038/clpt.1985.51

19. Wong C, Kumar SS, Graham GG, Begg EJ, Chin PK, Brett J, Ray JE, Marriott DJ, Williams KM, Day RO (2013) Comparing dose prediction software used to manage gentamicin dosing. Intern Med J 43(5): 519-525. doi:10.1111/imj.12067

20. Zhang Y, Huo M, Zhou J, Xie S (2010) PKSolver: An add-in program for pharmacokinetic and pharmacodynamic data analysis in Microsoft Excel. Comput Methods Prog Biomed 99(3):306-314. doi:10.1016/j.cmpb.2010.01.007

21. Sawchuk RJ, Zaske DE, Cipolle RJ, Wargin WA, Strate RG (1977) Kinetic model for gentamicin dosing with the use of individual patient parameters. Clin Pharmacol Ther 21(3):362-369

22. Fuchs A, Csajka C, Thoma Y, Buclin T, Widmer N (2013) Benchmarking therapeutic drug monitoring software: a review of available computer tools. Clin Pharmacokinet 52(1):9-22. doi:10. 1007/s40262-012-0020-y 